Supporting Information

\title{
Highly Stable Organic Antimony Halide Crystals for X-ray Scintillation
}

Qingquan He, ${ }^{\dagger}$ Chenkun Zhou, ${ }^{\dagger}$ Liangjin Xu,$^{\dagger}$ Sujin Lee, ${ }^{\dagger}$ Xinsong Lin,$^{\dagger}$ Jennifer Neu, ${ }^{\S}$ Michael

Worku, ${ }^{\mathbb{P}}$ Maya Chaaban, ${ }^{\dagger}$ and Biwu Ma ${ }^{*+,+, \mathbb{P}}$

†Department of Chemistry and Biochemistry, Florida State University, Tallahassee, FL 32306, USA

Department of Chemical and Biomedical Engineering, FAMU-FSU College of Engineering, Tallahassee, FL 32310, USA

${ }^{\S}$ National High Magnetic Field Laboratory, Florida State University, Tallahassee, Florida 32310, USA

PMaterials Science and Engineering Program, Florida State University, Tallahassee, FL 32306, USA

AUTHOR INFORMATION

Corresponding Author

*E-mail: bma@fsu.edu. 


\section{Experimental Section}

\section{Materials:}

Antimony (III) chloride $\left(\mathrm{SbCl}_{3}, 99.95 \%\right)$, bis(triphenylphosphoranylidene)ammonium chloride (PPNCl, 97\%), and Dichloromethane (DCM, $\geqslant 99.8 \%)$ were purchased from Sigma-Aldrich. Dimethylformamide (DMF, $99.8 \%$ ) and diethyl ether ( $\mathrm{Et}_{2} \mathrm{O}$, anhydrous) were bought for VWR.

\section{Growth of $(\mathrm{PPN})_{2} \mathrm{SbCl}_{5}$ and PPNCl single crystals:}

PPNCl $(1.14 \mathrm{mmol})$ and $\mathrm{SbCl}_{3}(0.57 \mathrm{mmol})$ were mixed at 2:1 molar ratio and dissolved in $4 \mathrm{~mL}$ DCM to form a clear precursor solution. $(\mathrm{PPN})_{2} \mathrm{SbCl}_{5}$ single crystals were prepared by injecting $0.9 \mathrm{~mL} \mathrm{Et}_{2} \mathrm{O}$ to $0.5 \mathrm{~mL}$ as-prepared precursor solution at room temperature in the $\mathrm{N}_{2}$-filled glovebox and stand for overnight. The large light color crystals were washed with $\mathrm{Et}_{2} \mathrm{O}$ and dried under reduced pressure. The preparation of PPNCl single crystals follows above procedures without adding $\mathrm{SbCl}_{3}$.

\section{Characterization:}

Single crystal X-ray diffraction (SCXRD) data of $(\mathrm{PPN})_{2} \mathrm{SbCl}_{5}$ was collected by using a Rigaku XtaLAB Synergy-S single crystal X-ray diffractometer with Mo $K \alpha$ radiation. The crystal was mounted in a cryoloop under Paratone-N oil and cooled to $150 \mathrm{~K}$ with an Oxford Cryosystem 800. A crystallographic information file (CIF) of (PPN) ${ }_{2} \mathrm{SbCl}_{5}$ has been deposited with CCDC (No. 1983725). Powder XRD spectrum of $(\mathrm{PPN})_{2} \mathrm{SbCl}_{5}$ was measured on a Rigaku SmartLab with copper X-ray tube radiation at a voltage of $40 \mathrm{kV}$ and $40 \mathrm{~mA}$. Simulated XRD pattern of $(\mathrm{PPN})_{2} \mathrm{SbCl}_{5}$ was calculated by Mercury software based on its crystallographic information file (CIF). Thermogravimetric analysis (TGA) was measured by using a TA Instruments TGA 550 system. The samples were heated from room temperature to $700{ }^{\circ} \mathrm{C}$ at a rate of $20{ }^{\circ} \mathrm{C} \cdot \mathrm{min}^{-1}$ in an argon atmosphere. UV-Vis absorption spectrum was carried out using a CARY 5000 UV-Vis NIR spectrophotometer (Agilent Technologies). 
Excitation, steady-state photoluminescence (PL), and Time-resolved PL (TRPL) spectra of $(\mathrm{PPN})_{2} \mathrm{SbCl}_{5}$ were measured on an Edinburgh FS5 spectrofluorometer (Edinburgh Instruments). For TRPL measurement, the excitation was provided by an Edinburgh EPLED-365 picosecond pulsed light emitting diode laser. The decay lifetime was calculated by monoexponential function $y=A_{1} \times \exp \left(-x / \tau_{1}\right)+y_{0}$. The PL quantum efficiencies (PLQEs) were acquired by using a Hamamatsu Quantaurus-QY Spectrometer equipped with a $150 \mathrm{~W}$ xenon lamp, calibrated integrating sphere. The PLQEs were calculated by the equation: $\eta_{Q E}=I_{S}\left(E_{R}-E_{S}\right)$, in which IS represents the luminescence emission spectrum of sample, $E_{R}$ is the spectrum of the excitation light from the empty integrated sphere, and $E_{s}$ is the excitation spectrum for exciting the sample.

Radioluminescence spectra were measured with an Edinburgh FS5 spectrofluorometer equipped with a 4 watts Mini-X2 X-ray tube (AMPTEK, Inc.). The radioluminescence spectra were collected by R928P photomultiplier with the spectral coverage from $200 \mathrm{~nm}$ to $870 \mathrm{~nm}$ equipped in the spectrofluorometer. The beam size of X-ray radiation source with $2 \mathrm{~mm}$ diameter is controlled by a brass collimator which consist with an aluminum insert and a cover that screws into X-ray tube. The dose rate of the X-ray tube under different power was detected by a Victoreen 450P-SI chamber survey radiation meter (see Figure S8 in Supporting Information). The crystal size of $(\mathrm{PPN})_{2} \mathrm{SbCl}_{5}$ which used for scintillator characterizations is $\sim 2.5 \times 3.0 \times 3.0 \mathrm{~mm}$. The distance between $\mathrm{X}$-ray tube and scintillator is $\sim 1 \mathrm{~cm}$. The detection limit was derived as the dose rate when the signal-to-noise ratio is 3 . As a reference, the commercial thallium activated cesium iodide $(\mathrm{CsI}(\mathrm{Tl}))$ scintillator with a thickness of $2.5 \mathrm{~mm}$ was purchased from Jiaxing AOSITE Photonics Technology Co., Ltd. and measured under the same conditions with ( $\mathrm{PPN})_{2} \mathrm{SbCl}_{5}$. 
Table S1. Single crystal XRD data and the corresponding collection parameters of $(\mathrm{PPN})_{2} \mathrm{SbCl}_{5}$. The data was recorded at a temperature of $150 \mathrm{~K}$.

\section{Compound}

Empirical formula

Formula weight

Temperature/K

Crystal system

Space group

$\mathrm{a} / \AA$

$\mathrm{b} / \AA$

$\mathrm{c} / \AA$

$\alpha /^{\circ}$

$\beta /{ }^{\circ}$

$\gamma /{ }^{\circ}$

Volume/ $\AA^{3}$

Z

$\rho_{\text {calc }} \mathrm{g} / \mathrm{cm}^{3}$

$\mu / \mathrm{mm}^{-1}$

Crystal size $/ \mathrm{mm}^{3}$

Radiation

$2 \Theta$ range for data collection ${ }^{\circ}$

Index ranges

Reflections collected

Independent reflections

Data/restraints/parameters

Goodness-of-fit on $\mathrm{F}^{2}$

Final $R$ indexes $[\mathrm{I}>=2 \sigma(\mathrm{I})]$

Final $\mathrm{R}$ indexes [all data]

Largest diff. peak/hole / e $\AA^{-3}$
Antimony

pentachloride

bis(triphenylphosphoranylidene)ammonium

$\mathrm{C}_{72} \mathrm{H}_{60} \mathrm{~N}_{2} \mathrm{P}_{4} \mathrm{SbCl}_{5}$

1376.10

$149.99(10)$

monoclinic

$\mathrm{P} 2{ }_{1} / \mathrm{c}$

$11.0421(2)$

24.1416(4)

24.0743(4)

90

90.6060(10)

90

6417.22(19)

4

1.424

0.783

$0.303 \times 0.264 \times 0.128$

$\operatorname{Mo~} \operatorname{K} \alpha(\lambda=0.71073)$

3.688 to 65.202

$-15 \leq \mathrm{h} \leq 14,-34 \leq \mathrm{k} \leq 32,-32 \leq 1 \leq 36$

92625

$19486\left[\mathrm{R}_{\text {int }}=0.0524, \mathrm{R}_{\text {sigma }}=0.0424\right]$

19486/0/757

1.086

$\mathrm{R}_{1}=0.0346, \mathrm{wR}_{2}=0.0840$

$\mathrm{R}_{1}=0.0470, \mathrm{wR}_{2}=0.0881$

$0.60 /-0.68$ 
Table S2. Selected bond lengths and bond angles of $(\mathrm{PPN})_{2} \mathrm{SbCl}_{5}$.

\begin{tabular}{lllllll}
\hline Atom & Atom & Length $/ \AA$ & Atom & Atom & Atom & Angle ${ }^{\circ}$ \\
\hline $\mathrm{Sb} 1$ & $\mathrm{Cl1}$ & $2.3810(4)$ & $\mathrm{Cl1}$ & $\mathrm{Sb} 1$ & $\mathrm{Cl} 2$ & $87.908(19)$ \\
$\mathrm{Sb} 1$ & $\mathrm{Cl} 2$ & $2.6397(5)$ & $\mathrm{Cl1}$ & $\mathrm{Sb} 1$ & $\mathrm{Cl} 3$ & $89.713(16)$ \\
$\mathrm{Sb} 1$ & $\mathrm{Cl3}$ & $2.5964(5)$ & $\mathrm{Cl1}$ & $\mathrm{Sb} 1$ & $\mathrm{Cl} 4$ & $87.314(16)$ \\
$\mathrm{Sb} 1$ & $\mathrm{Cl4}$ & $2.5876(4)$ & $\mathrm{Cl1}$ & $\mathrm{Sb} 1$ & $\mathrm{Cl} 5$ & $88.669(15)$ \\
$\mathrm{Sb} 1$ & $\mathrm{Cl5}$ & $2.6363(5)$ & $\mathrm{Cl} 3$ & $\mathrm{Sb} 1$ & $\mathrm{Cl} 2$ & $91.274(19)$ \\
& & & $\mathrm{Cl} 3$ & $\mathrm{Sb} 1$ & $\mathrm{Cl} 15$ & $177.896(15)$ \\
& & & $\mathrm{Cl} 14$ & $\mathrm{Sb} 1$ & $\mathrm{Cl} 2$ & $175.209(19)$ \\
& & & $\mathrm{Cl} 14$ & $\mathrm{Sb} 1$ & $\mathrm{Cl} 3$ & $89.059(15)$ \\
\hline
\end{tabular}

(a)

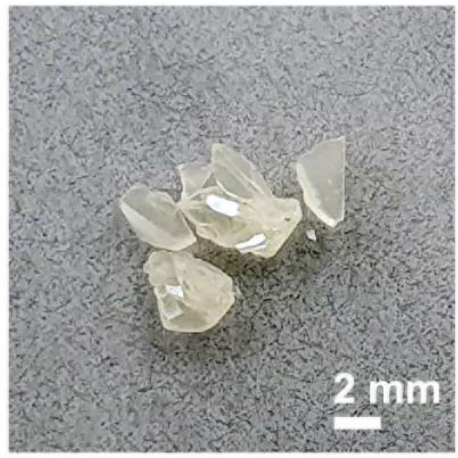

(b)

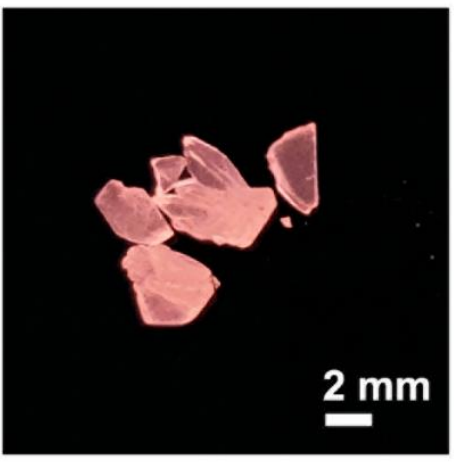

Figure S1. Digital photographs of $(\mathrm{PPN})_{2} \mathrm{SbCl}_{5}$ single crystals under (a) ambient light and (b) UV light (365 nm). 


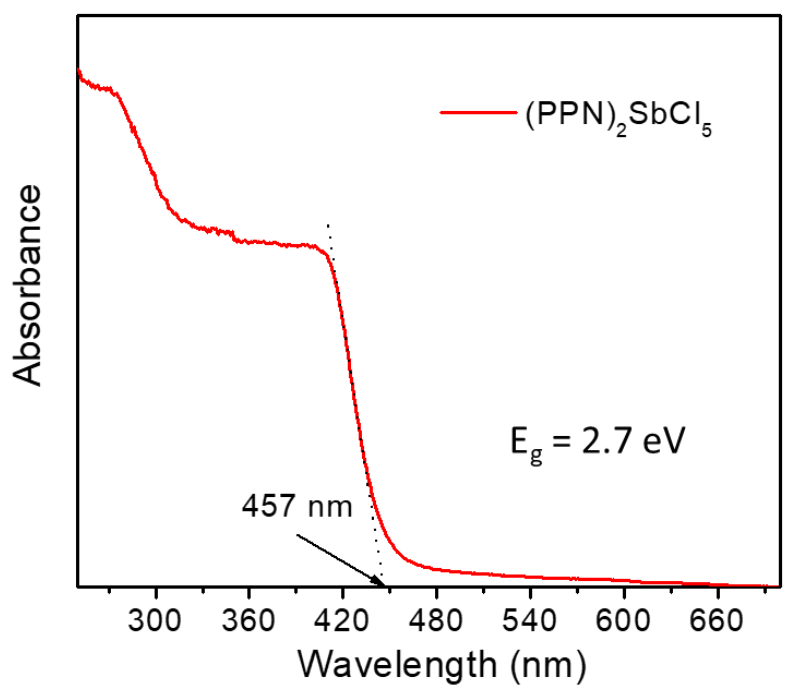

Figure S2. UV-Vis absorption spectrum of $(\mathrm{PPN})_{2} \mathrm{SbCl}_{5}$.

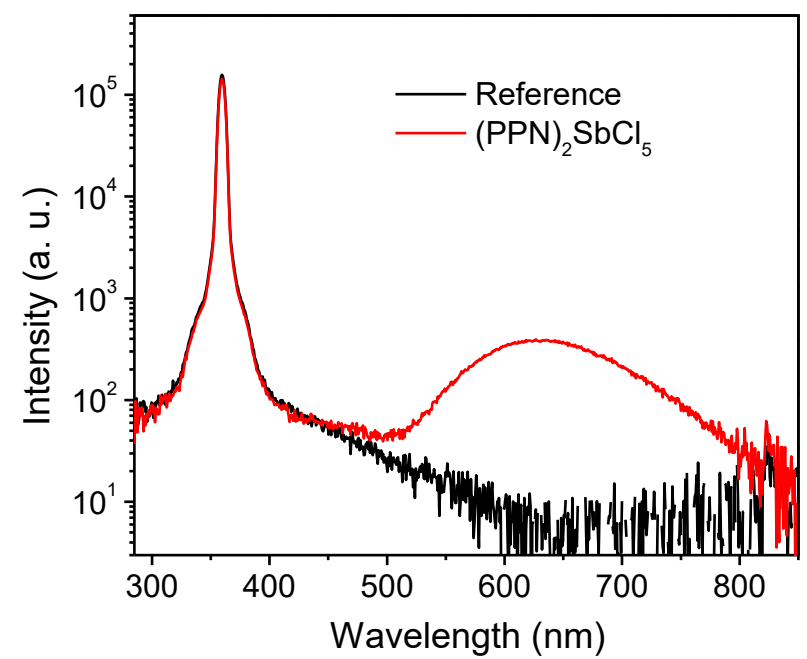

Figure S3. Excitation line of reference and emission spectrum of $(\mathrm{PPN})_{2} \mathrm{SbCl}_{5}$ collected by an integrating sphere. The PLQE was calculated by the equation: $\eta_{Q E}=I_{S}\left(E_{R}-E_{S}\right)$. 


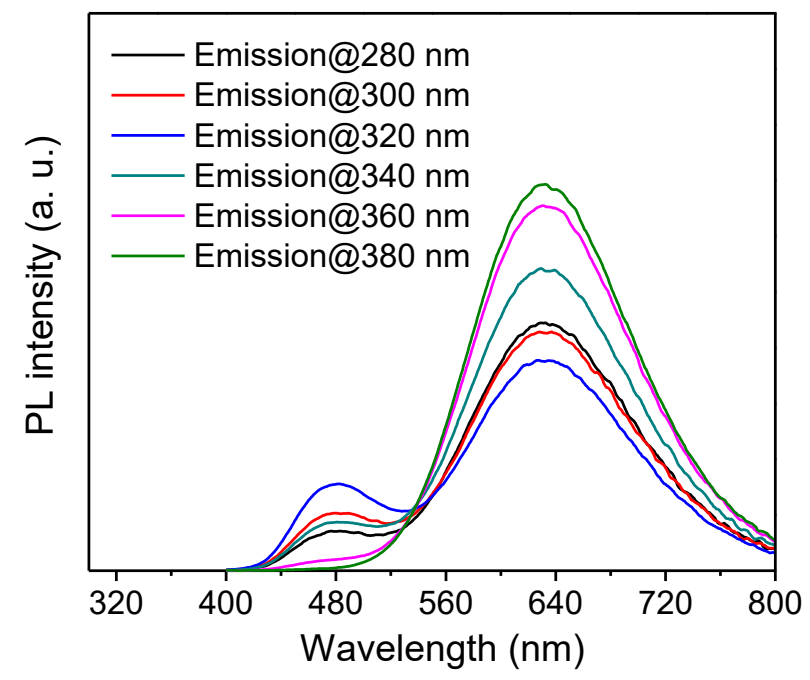

Figure S4. PL spectra dependent on excitation wavelength from 280 to $380 \mathrm{~nm}$ of (PPN)$)_{2} \mathrm{SbCl}_{5} \mathrm{crystals}_{\text {. }}$

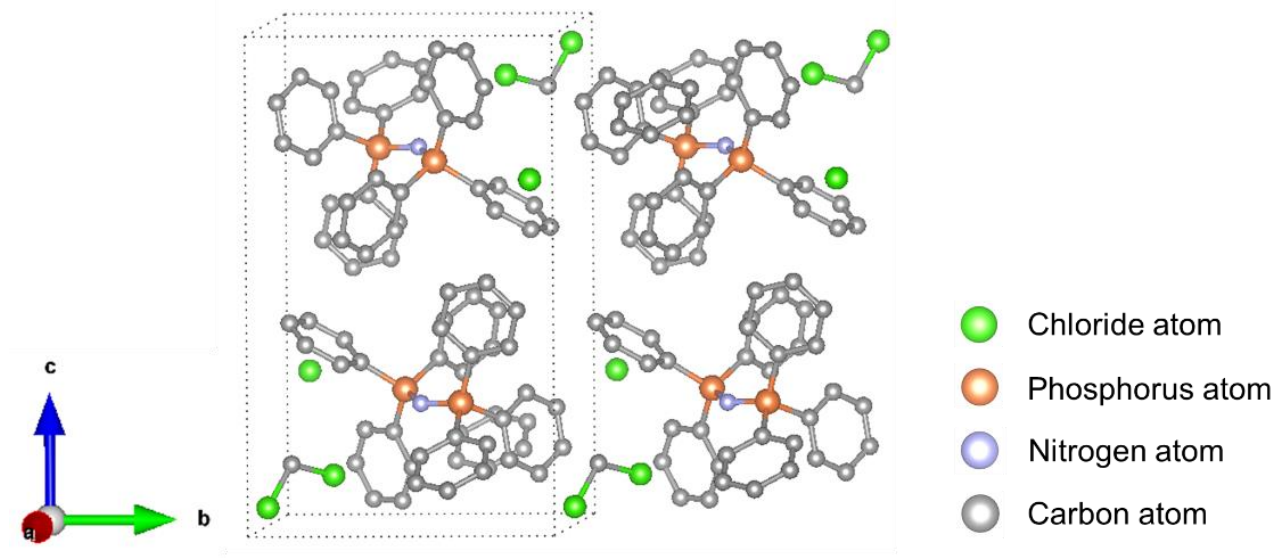

Figure S5. Single crystal structure of PPNCl. 

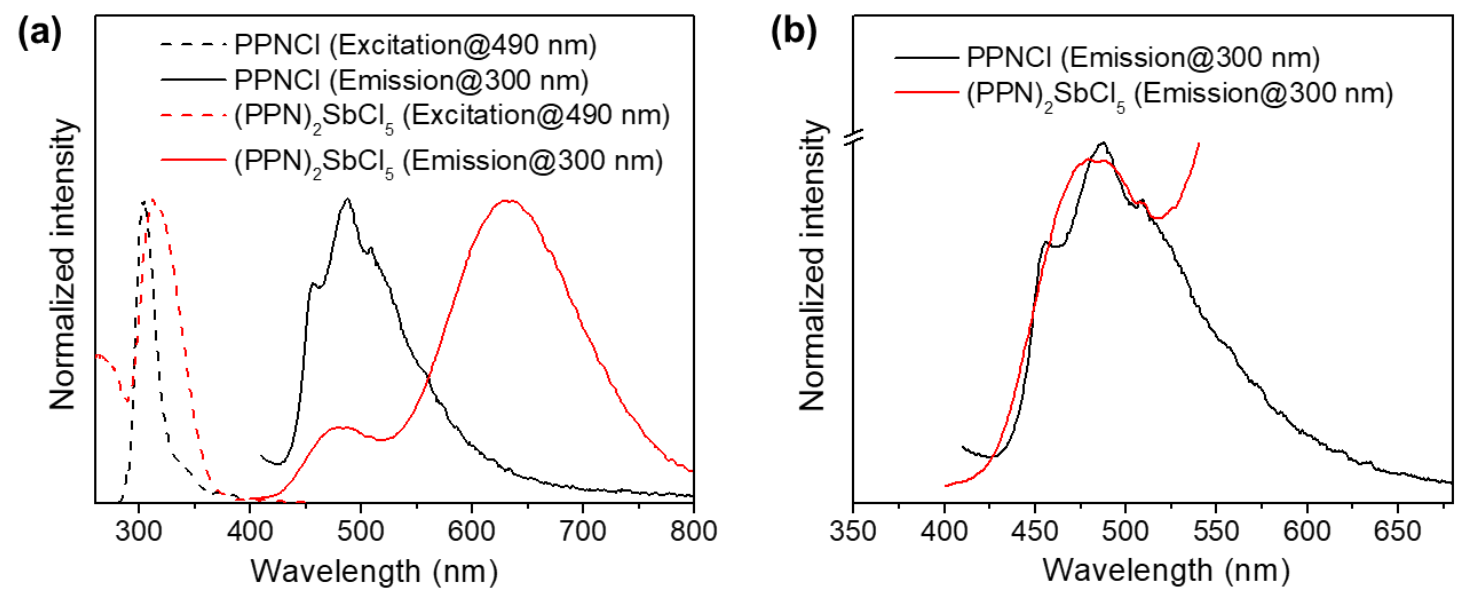

Figure S6. (a) Excitation and emission spectra of $\mathrm{PPNCl}$ and $(\mathrm{PPN})_{2} \mathrm{SbCl}_{5}$ single crystals. (b) The comparison of spectra of $\mathrm{PPNCl}$ and $(\mathrm{PPN})_{2} \mathrm{SbCl}_{5}$ at cyan region.

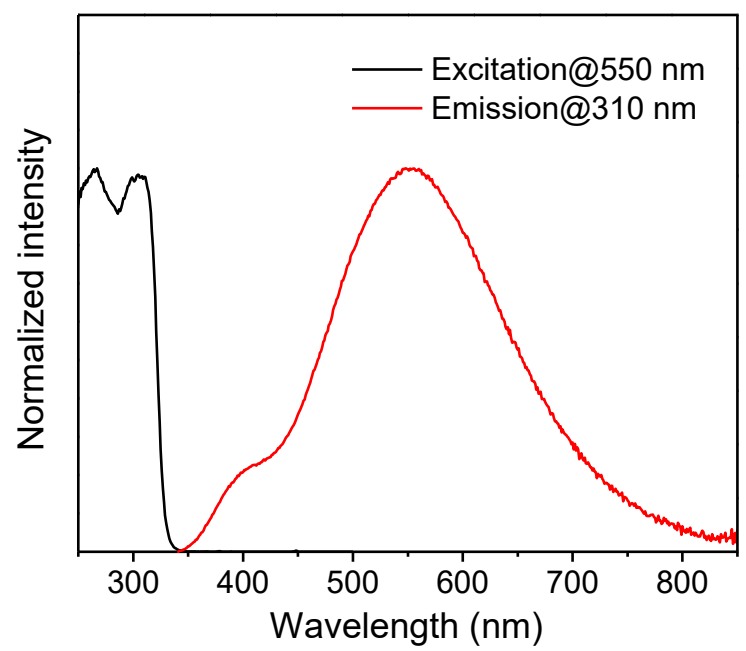

Figure S7. Excitation and emission spectra of CsI(Tl) crystals. 


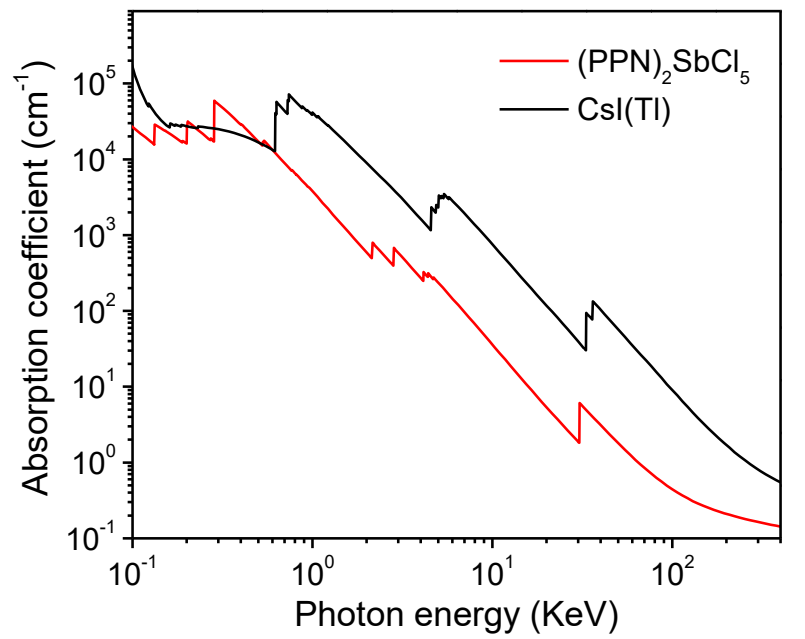

Figure S8. The X-ray absorption coefficients of $(\mathrm{PPN})_{2} \mathrm{SbCl}_{5}$ and $\mathrm{CsI}(\mathrm{Tl})$ as a function of photon energy. The data was obtained from National Institute of Standards and Technology (https://physics.nist.gov/PhysRefData/FFast/html/form.html).

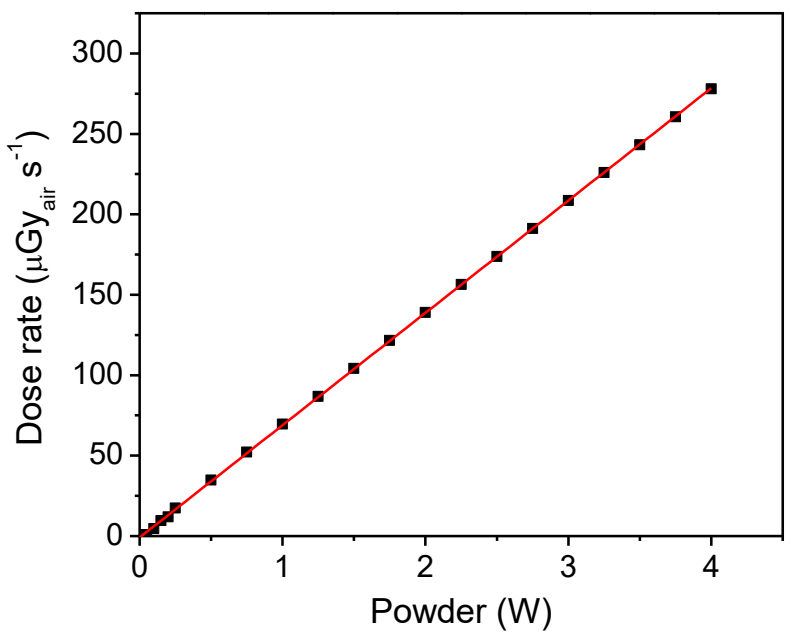

Figure S9. X-ray dose rate versus power for Mini-X2 X-ray tube (AMPTEK, Inc.). 

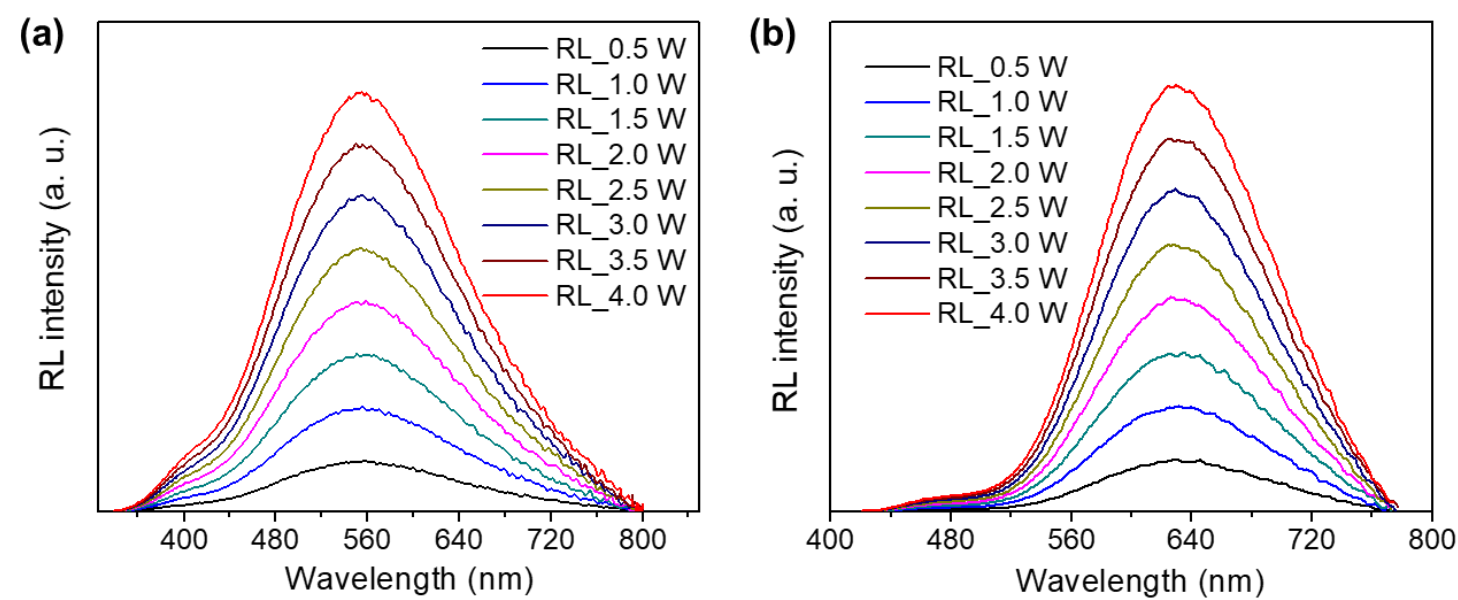

Figure S10. RL spectra of (a) $\mathrm{CsI}(\mathrm{Tl})$ and (b) $(\mathrm{PPN})_{2} \mathrm{SbCl}_{5}$ under X-ray irradiation with tube powder from 0.5 to $4 \mathrm{~W}$.

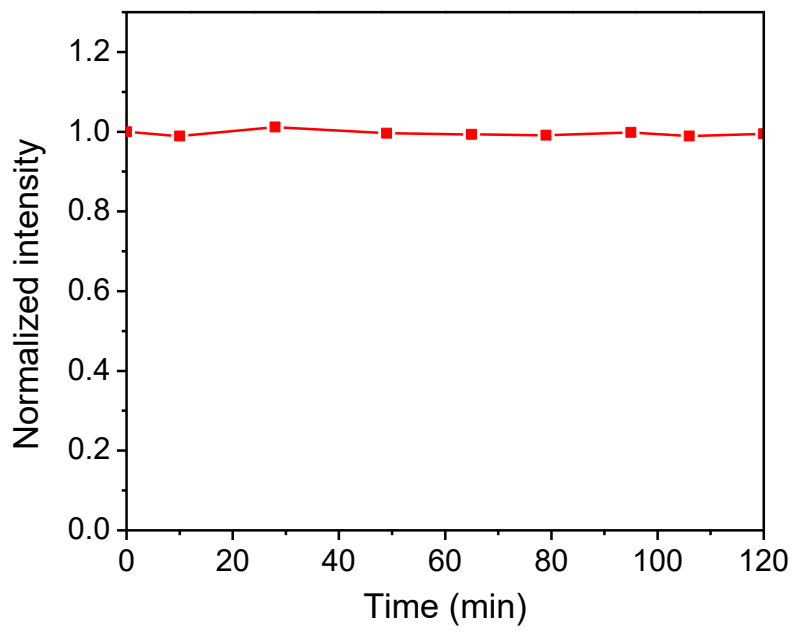

Figure S11. PL stability of $(\mathrm{PPN})_{2} \mathrm{SbCl}_{5}$ crystals under continuous illumination using a high-power mercury $\operatorname{lamp}\left(150 \mathrm{~mW} \mathrm{~cm}^{-2}\right)$. 